\title{
hTERT Suppression via Small Interference RNA in Cervical Cancer Cells
}

\author{
Shawn Gray*, Douglas Christensen \\ Department of Life Sciences, Wayne State College, Wayne, NE \\ Student:shgray01@,outlook.com* \\ Mentor:dochris1@,wsc.edu
}

\begin{abstract}
Telomerase (TERT) functions to extend the telomeric repeat terminus of each chromosome allowing embryonic cells to proliferate into an adult organism. The TERT gene is subsequently inactivated following maturation, consequently conveying a finite lifespan to every adult cell line, as shortened chromosome arms trigger cell apoptosis. This process ensures that older cells lines, which are invariably accumulating mutations, are eliminated from the body and replaced by stem cells containing founding DNA. One of the defining attributes of a cancer cell is the ability to divide perpetually. This capability to divide continually is often due to the reactivation of TERT. Therefore, the abolishment of TERT activity presents a promising avenue for cancer treatment. Here, we demonstrate through qRT-PCR and ELISA techniques that although small interference RNA (siRNA) results in a transcription knockdown of ninety-seven percent the actual protein activity reduction is far less dramatic.
\end{abstract}

\section{KEYWORDS}

Telomerase; Small Interference RNA; Cancer; Tumor; hTERT; qRT-PCR; RNA Interference; ELISA

\section{INTRODUCTION}

Telomerase (TERT) is one of the many constituent proteins comprising an embryonic cells enzyme pool. TERTs function is to extend the simple telomeric repeat sequence fastened to the end of each chromosome. ${ }^{1}$ This sequence operates as a molecular timer, counting down to the exact time of apoptosis (typically forty to fifty divisions). TERT activity is of paramount importance to a developing embryo, by elongating the telomeric sequence, TERT obligates a cell to divide indefinitely. Thusly, TERT activity induces an embryo to proliferate into the trillions of cells that make up the adult organism. The TERT gene is subsequently inactivated following maturation, consequently conveying a finite lifespan to every adult cell line. ${ }^{1}$ By allowing each cell only a limited amount of environmental exposure, the body is able to impede the amplification of mutations that may engender a diseased state. One of the defining attributes of a cancer cell is immortality, or the ability to divide perpetually. This unique capability is due to the reactivation of TERT. Therefore, the abolishment of TERT activity presents a promising avenue for cancer treatment as TERT specific targeting may allow for a dramatic decrease in the side effects associated with many of the current cancer therapies.

Cancer, in the simplest description, is unregulated cell division. The sustained proliferation of a cancer cell will ultimately spawn a distended mass of daughter cells recognized as a tumor. The biochemical mechanisms that induce a cancerous state are invariably complex and can occur through many disparate routes. Nevertheless, there are six specific characteristics a cell must possess in order to be considered malignant. ${ }^{1}$ The six attributes include constant divisional signaling, unresponsiveness to growth suppressors, apoptotic resistance, the ability to induce angiogenesis, the ability to metastasize, and finally replicative immortality. Individually, these abnormal faculties would not breed a cancerous state, but when occurring concomitantly they confer an unrelenting proliferative capacity to whichever cell acquires them. Of the six requisite characteristics, replicative immortality is targeted in this research. This particular characteristic is greatly influenced by the Telomerase protein, the function of which is described below. The majority of normal somatic cells possess a finite replicative potential. This limited reproductive ability can be attributed to a eukaryotic cell's linear chromosomes and the "end replication problem" this linearity confers. ${ }^{2}$ In order for the DNA replicative machinery to form novel strands, short RNA primers are employed, these primers are eventually supplanted with DNA. However, due to the aforementioned chromosomal uniformity the RNA primer located at the 5' end of the novel strand cannot be replaced and disintegrates shortly following S phase. Consequently, a small segment of chromosomal DNA is forsaken during each mitotic event. The situation described hitherto is known as the "end replication problem" and (by limiting a cells lifespan) has been hypothesized to reduce the amount of mutational damage a cell will endure. ${ }^{3}$ This constraint will also act to curb the probability of a mutation based disease arising from the cell, which is advantageous to the organism as a whole.

The DNA squandered during each divisional sequence however, is not protein encoding, it is simply a component of a repetitive sequence known as the telomere. For eukaryotes, this sequence is most often 5'-T'TAGGG-3'. The telomere acts as a molecular clock buffering the degenerative action of mitosis so as to confer to the cell a set number of potential divisions. ${ }^{2}$ All though apoptosis is a potential result of telomere shortening, Olovnikov demonstrates senescence as another possibility. ${ }^{3}$ Senescent cells do not undergo apoptosis, yet they do not continue to divide either. Senescence is rather a state of suspended animation in which 
the cell cycle perpetually rests in the G1 phase. Eventually, these languid cells initiate, and are subsequently cleared by an innate immune response. ${ }^{4}$ Nonetheless whether a cell undergoes apoptotic death or cell cycle arrest in the form of senescence, the end result with respect to cancer is the same; the cell is unable to contribute to tumor development. The actual mechanics by which shortened telomeres initiate senescence and apoptosis are complex and not fully understood. However, current literature from Blasco, Saretzki and colleagues as well as Janknecht has yielded some information about this process and it is almost certain more will be gathered. $5,6,7 \mathrm{As}$ is stands, however, both senescence and the potential of crisis leading to apoptosis is thought to only lessen the likelihood of cancer. In order for a cancer cell to have any prospect of reaching immortality, it must circumvent the crisis cascade.

Crisis evasion can only be attained by lengthening the telomere, which can only occur via the activity of telomerase. Consequently, the sole avenue by which a cancer cell can acquire immortality is through telomerase reactivation. ${ }^{2}$ Ninety percent of all characterized cancers achieve telomere elongation through the reactivation of human Telomerase (hTERT). Although some lowfrequency cancers do not appear to adhere to this mechanism. ${ }^{8}$ The specific mechanism by which these cells lengthen their telomeric repeat sequence has not been delineated and is hypothesized to occur through an ALT or alternative telomere lengthening mechanism. The ALT mechanism will conceivably employ a plethora of proteins that could probably be targeted by the same mechanism employed for hTERT degradation (described below). The use of organic inhibitors is one potential method by which protein activity, such as hTERT, can be inhibited. Organic inhibitor specifics and delivery methods can vary a great deal. The medical industry already employs a multitude of disparate materials to achieve protein degradation and/or inhibition. For example, many small organic molecules have proven useful in inactivating a given enzyme by either competitive or allosteric inhibition. An additional advantage of these organic materials is their ability to elicit enzymatic inactivation without the side effect of generating an immune response (due to their small size). ${ }^{9}$ Unfortunately, no matter how advantageous these minuscule particles are, accompanying them are at least a few undesirable side effects due to their low protein binding specificity. Many of the particles appropriated to treat enzyme disorders not only inhibit their target enzyme but a number of closely related protein catalysts. A much more specific degradation mechanism, microRNA, is already being utilized by many eukaryotic cells as a means of translational control.

MicroRNAs are $\sim 22$ nucleotides long, endogenously synthesized RNA particles encoded by specific regulatory genes. These microRNA fragments are never translated but instead act to inhibit mRNA translation by binding specific base pair sequences of protein producing mRNA strands and extorting their deterioration. ${ }^{10}$ Another more therapeutically useful form of RNA interference employs externally originating small interfering RNA (siRNA). Initially, an extended, double stranded segment of RNA is transformed into the target cell. Subsequently, the dsRNA is circumscribed by a cytoplasmic protein called Dicer. Dicer cleaves specific segments of dsRNA effectively releasing a 21-23 nucleotide fragment that possesses two nucleotide 3' overhangs at each terminus, these overhangs are important for downstream protein interactions. Traditionally, therapeutic RNA forgoes the Dicer modification by introducing an RNA fragment of 23 nucleotides with previously synthesized 3' overhangs. By directly inserting this preformed siRNA a researcher can ensure that the desired sequence is in fact incorporated into the interference machinery. The Dicer derived 21-23 nucleotide fragments are then incorporated into an immense protein complex known as RISC or RNA induced silencing complex. ${ }^{11}$ A constituent RISC protein called Argonaute-2 (Ago 2) binds the siRNA at its 3' overhang and removes one of the complementary strands. The resulting single stranded RNA segment (still within the RISC complex) can then bind a specific sequence in an mRNA (usually in the 3' untranslated region). Upon binding, the RISC complex will cleave the mRNA in two, the resulting messenger RNA fragments are subsequently degraded by cytoplasmic nucleases. Either strand may be incorporated into the complex during RISC formation. The thermodynamic stability of the siRNA's 5' end dictates which strand will be assimilated. ${ }^{12}$ Consequently, a researcher is capable of definitively dictating which siRNA strand will be expressed on the RISC complex and in turn the specific mRNA that will be targeted for degradation (hTERT in this research). The fact that siRNA is of external origin and must, therefore, be incorporated into the cytoplasm of a cell from the surrounding environment introduces a formidable complication to the previously discussed approach. There are several mechanisms by which this transfection can be accomplished; the one employed in this research is a nanoparticle.

Many disparate nanoparticle classes exist; the one employed in this research is an MPG based peptide-RNA complex where the MPG proteins form stable, non-covalent bonds with the siRNA, typically in a 15:1 ratio. The resulting 15:1 amphipathic nanoparticle can subsequently interact with proteoglycans in a cell membrane. This collaboration induces actin rearrangements through a Rac-1 intermediate. The actin disruptions initiate the formation of ephemeral $\beta$-barrel like pores that allow the nanoparticle to diffuse through the plasmalemma and effectively enter the cells cytoplasm independent of phagocytosis. ${ }^{13}$ Consequently, the siRNA is also able to avoid lysosomal degradation. ${ }^{14}$ Once this particle enters the target cells cytoplasm it must discharge its nucleotide patron to the surrounding environment. This is accomplished by the overall negative charge within the cell. When the nanoparticle enters the negative cytoplasmic environment, the positive portion of the MPG protein begins to interact with many of the negatively charged compounds within the cell. As these interactions increase, the MPG peptide bonds with the nucleotide particle will begin to weaken until eventually the siRNA is released. 
The dominant inquiry of this research is whether or not siRNA can be effectively transfected into cancer cells and if that siRNA can subsequently elicit the degradation of hTERT mRNA. Furthermore, if hTERT mRNA degradation is achieved, what are the immediate consequences on hTERT protein accumulation? The relative quantification of hTERT mRNA from siRNA treated and untreated HeLa cell groups, via quantitative reverse transcriptase polymerase chain reaction (qRT-PCR), will yield a definitive answer to this first question while Enzyme Linked Immunosorbent Assays (ELISA) will shed light on the actual effect on hTERT proteins. The hypothesis of this procedure was as follows: siRNA can elicit the degradation of hTERT mRNA in cultured HeLa cells and this degradation will significantly reduce hTERT protein concentrations. Thus, this method could potentially be employed as an in vivo cancer treatment. Much research concerning siRNA and its knockdown effect on Htert mRNA has already been published, however research regarding how this treatment affects the protein itself is significantly scarcer. This research will not only demonstrate the effect siRNA has on hTERT mRNA but also how this knockdown correlates with protein degradation.

\section{METHODS AND PROCEDURES}

\section{Cell Culture}

HeLa (ATCC® CCL-2) cells $(1.5 \mathrm{~mL})$ were rapidly defrosted in a $37^{\circ} \mathrm{C}$ water bath and deposited into a $250 \mathrm{~mL}$ Fisher ${ }^{\mathrm{TM}}$ brand cell culture flask containing 10\% v/v Fetal Bovine Serum (ATCC), 1\% v/v Pen Strep and Delbecco's Modified Eagle Medium (DMEM) previously vacuum filtered for sterility prior to incubation at $37^{\circ} \mathrm{C}$ in $5 \% \mathrm{CO}_{2}$. Confluence level cells were displaced via Life Technologies TrypLE ${ }^{\mathrm{TM}}$ Express Enzyme, and once more grown to confluence. Additionally, confluence level HeLa cells were simultaneously developed in each well of two Fisher ${ }^{\mathrm{TM}}$ brand 6 well plates. Media containing Sigma-Aldrich N-TER Nanoparticle siRNA Transfection System ${ }^{\mathrm{TM}}$ was utilized in all six wells of one aforementioned plate per manufacturer's instruction (minus positive control). The same solution, minus the nanoparticle, was also instilled in the six remaining control wells. All cell inoculations were conducted in a UV sterilized laminar flow hood.

\section{$m \mathrm{RN} A$ and Protein Isolation}

The siRNA sequence exploited in this inquiry was designed by the researcher to complement two divergent regions of hTERT mRNA following the protocol developed by Reynolds and colleagues in 2004, these sequences are as follows: 5'-

GAAGCCGAAGGCCAGCACGUU-3', 5'-CAUCAGCCAGUGCAGGAACUU-3'. ${ }^{15}$ Successively ensuing a 24 hour incubation period with the experimental nanoparticle solution, each sample's mRNA and protein was isolated via the Trizol ${ }^{\mathrm{TM}}$ (Life Technologies ${ }^{\mathrm{TM}}$ ) method to include a DNAse I (New England Biolabs ${ }^{\mathrm{TM}}$ ) treatment to eliminate possible DNA feedback during qRT-PCR analysis. Subsequently, three specific samples from each group (control and experimental) were chosen at random for qRT-PCR and ELISA analysis.

\section{$m \mathrm{RN} A$ Quantification}

Relative quantification of h'TERT mRNA was conducted via qRT-PCR utilizing an Illumina Eco ${ }^{\text {TM }}$ Real Time PCR System. A two-step procedure was conducted with a separate reverse transcription using New England Biolab's ${ }^{\text {TM }}$ AMV First Strand cDNA Synthesis Kit. Initially, $250 \mu \mathrm{L}$ of a PCR master mix (Bio-Rad Laboratories, Inc. ${ }^{\text {TM}}$ ), consisting of $2 \mathrm{X}$ qPCR reaction buffer, 4 mM $\mathrm{MgCl}_{2}, 0.4 \mathrm{mM}$ dNTP's, $0.0004 \mathrm{mM}$ forward primer and $0.0004 \mathrm{mM}$ reverse primer was generated for use in the final amplification. Beacon ${ }^{\mathrm{TM}}$ generated PCR primers (5'-TCCCTGCGT'TCT'TGGCTTTC -3' and 5'-CCTGCGCGTCATCTCTGA3') were designed to generate a $148 \mathrm{bp}$ long hTERT derived fragment. The primer sequence of the internal control gene, glyceraldehyde phosphate dehydrogenase (GAPDH), is: 5'-CCTGCACCACCAACTGCT'TAG-3', 5'-

TGAGTCCTTCCACGATACCAA-3'. qRT-PCR primer design was optimized through protocol published by Singh and Kumar (2001). ${ }^{16}$ qRT-PCR was conducted in an Illumina ${ }^{\text {TM }} 48$ well reaction plate. $14 \mu \mathrm{L}$ of the previously mentioned master mix was deposited into each designated well. Additionally, $6 \mu \mathrm{L}$ of cDNA sample (3 experimental and 3 control) was added to their proper

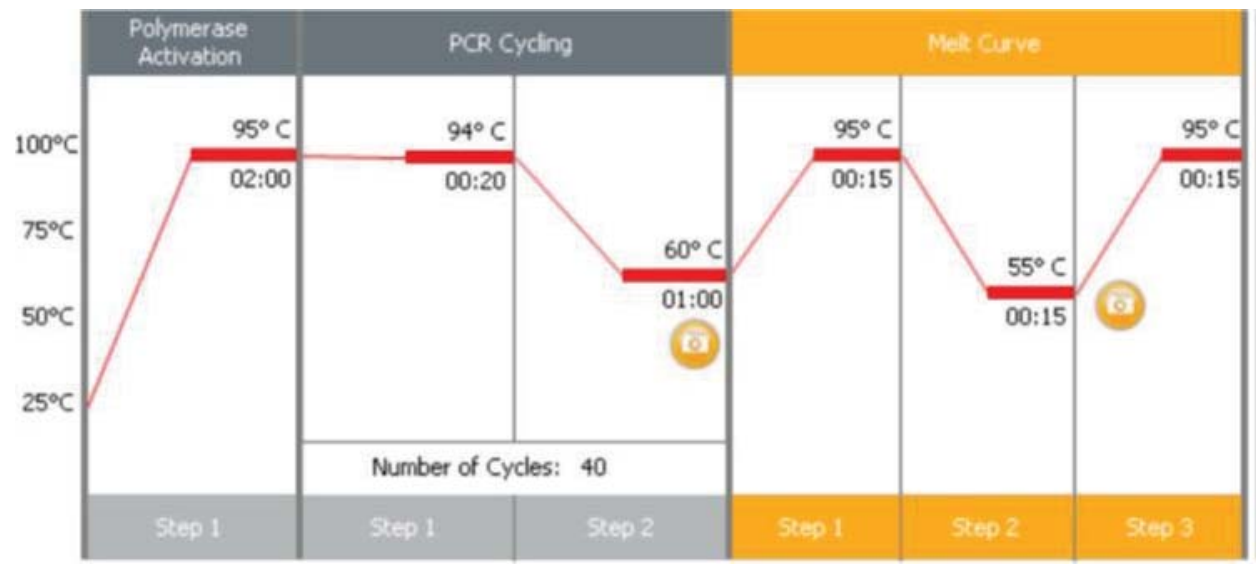

Figure 1. qRT-PCR thermal cycle diagram of format utilized for forty primer elongations. 
wells. One exception to this generalization can be found in the formation of the negative controls, each with either GAPDH or hTERT master mix and nuclease free water (no DNA sample). The overall mechanism underlying the PCR reaction itself is relatively simple. Initially, the PCR solution is warmed to $95^{\circ} \mathrm{C}$, at which point the cDNA fragments dissociate allowing primer binding. Next, the solution is cooled to $55^{\circ} \mathrm{C}$, allowing both primer binding and TaqC polymerase to catalyze primer elongation (Figure 1).

\section{qPCR Normalization}

In order to account for inevitable cell count differences between incubation wells, Glyceraldehyde phosphate dehydrogenase, was analyzed to normalize the data allowing a valid comparison between cell samples regardless of variation in cell count. Depicted in Figure 2 is the actual qRT-PCR plate layout (Illumina ${ }^{\text {TM }} 48$ well) employed in this research. Each six square grouping in the figure corresponds to analyzed mRNA that was initially isolated from cells grown in one well of the aforementioned 6 well plates. From each of these biological replicates, six technical replicates were produced. Three of these technical replicates were analyzed for hTERT mRNA, while the remaining three were evaluated for the internal control gene, GAPDH. Blue squares indicate exposure to siRNA whereas green highlights correspond to control samples. The possibility of primer-dimers and non-target amplifications exists in any PCR reaction. To determine whether or not such an aberration materialized during the reaction, a melt curve was generated (Figure 4). The overall question of this procedure is how effective was the siRNA treatment at reducing the cells hTERT mRNA content. In order to address this, Cq value analysis was performed. Cq is the number of cycles required to reach a previously specified fluorescence threshold. The fewer cycles required to reach the threshold, the more cDNA fragments there were in the original sample. The Cq value for each technical replicate in a given sample was averaged. Following this calculation, the average GAPDH Cq was subtracted from that of the average hTERT value to generate one $\Delta$ Cq value for each

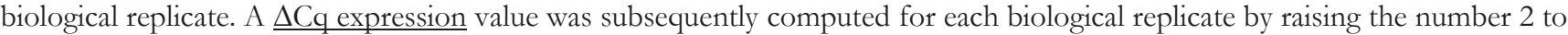
the negative $\Delta \mathrm{Cq}$ value. Next, the $\Delta \mathrm{Cq}$ expression level of each biological replicate, in a given treatment group, was averaged to beget 2 mean $\Delta \mathrm{Cq}$ expression levels for each analyzed group. The resulting mean $\Delta \mathrm{Cq}$ was divided by that of the control group. The resulting value is the $\Delta \Delta \mathrm{Cq}$ value for the experimental group while the control was given a reference number of 1 . The $\Delta \Delta \mathrm{Cq}$

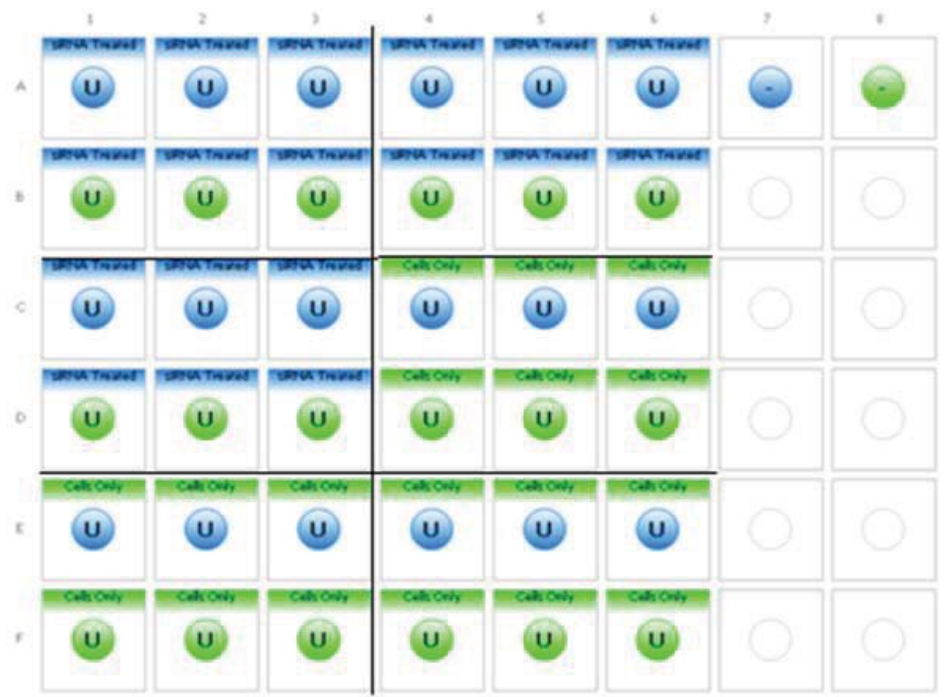

Figure 2. qRT-PCR plate layout, each six square grouping (separated by black lines) corresponds to analyzed mRNA initially isolated from cells grown in one well of a six well plate.

value of each group was subsequently used to generate a knockdown graph (Figure 5). All of the aforementioned mathematical analyses were conducted in an Excel spreadsheet following protocol published by Haimes and Kelley in $2013 .{ }^{17}$ 
ELIS A Analysis

Corresponding hTERT protein analysis was performed via an Enzyme Linked Immunosorbent Assay (ELISA) in a Fisher ${ }^{\mathrm{TM}}$ brand 96-well ELISA plate. A schematic representation of the ELISA plate layout is depicted in Figure 3. The three biological replicates from each experimental group (siRNA treated and control) were treated as described for the qRT-PCR samples above. TERT H-321 (cat \# 7212, Santa Cruz Biotech) and GAPDH FL-335 (Cat \# sc-25778, Santa Cruz Biotech) was utilized as primary antibody while the secondary antibody consisted of goat-anti rabbit IgG-HRP (cat \# sc-2004, Santa Cruz Biotech). Optimum protein concentrations were determined to be $10 \mu \mathrm{g} / \mathrm{mL}$ via criss-cross serial dilution assay. Each samples original protein concentration was ascertained through bicinchoninic acid assay (BCA) and subsequently diluted (in PBSN containing 137 $\mathrm{mM} \mathrm{NaCl}, 2.7 \mathrm{mM} \mathrm{KCl}, 4.3 \mathrm{mM} \mathrm{Na} \mathrm{HPO}_{4} \cdot 7 \mathrm{H}_{2} \mathrm{O}, 1.4 \mathrm{mM} \mathrm{KH} \mathrm{PO}_{4}$ and $0.05 \% \mathrm{w} / \mathrm{v} \mathrm{NaN}$ ) to this optimum ELISA concentration $(10 \mu \mathrm{g} / \mathrm{mL})$. Additionally, $50 \mu \mathrm{L}$ of protein sample was deposited into each respective well within the 96 -well ELISA plate and incubated overnight at room temperature. This plate was washed three separate times with deionized water, so as to remove any unbound proteins. A blocking buffer, consisting of $17 \mathrm{mM} \mathrm{Na} \mathrm{B}_{4} \mathrm{O}_{7} \cdot 10 \mathrm{H}_{2} \mathrm{O}, 120 \mathrm{mM} \mathrm{NaCl}, 0.05 \% \mathrm{v} / \mathrm{v}$ Tween 20, $1 \mathrm{mM}$ EDTA, $0.25 \% \mathrm{v} / \mathrm{v}$ Bovine Serum Albumin, and $0.05 \% \mathrm{w} / \mathrm{v}$ sodium azide, $\mathrm{pH}$ adjusted to $8.5 \mathrm{with} \mathrm{NaOH}$, was used to fill each well. This procedure was followed by an incubation period of 30 minutes at room temperature. Excess blocking buffer was removed by three DI water washes. $50 \mu \mathrm{L}$ of secondary antibody was then garnered into each well at a concentration of 50 $\mathrm{ng} / \mathrm{mL}$ in PBSN, as determined by criss-cross serial dilution. This solution was incubated for two hours at room temperature. The same DI water/ blocking buffer wash was performed as in the previous step at which point a final, secondary antibody solution of $50 \mu \mathrm{L}$ was employed. The concentration of this mixture was $250 \mathrm{ng} / \mathrm{mL}$. $100 \mu \mathrm{L}$ of TMB (tetramethylbenzidine) was added as the colorimetric substrate and analyzed at 450 nanometers on a microplate reader. This procedure was conducted following a

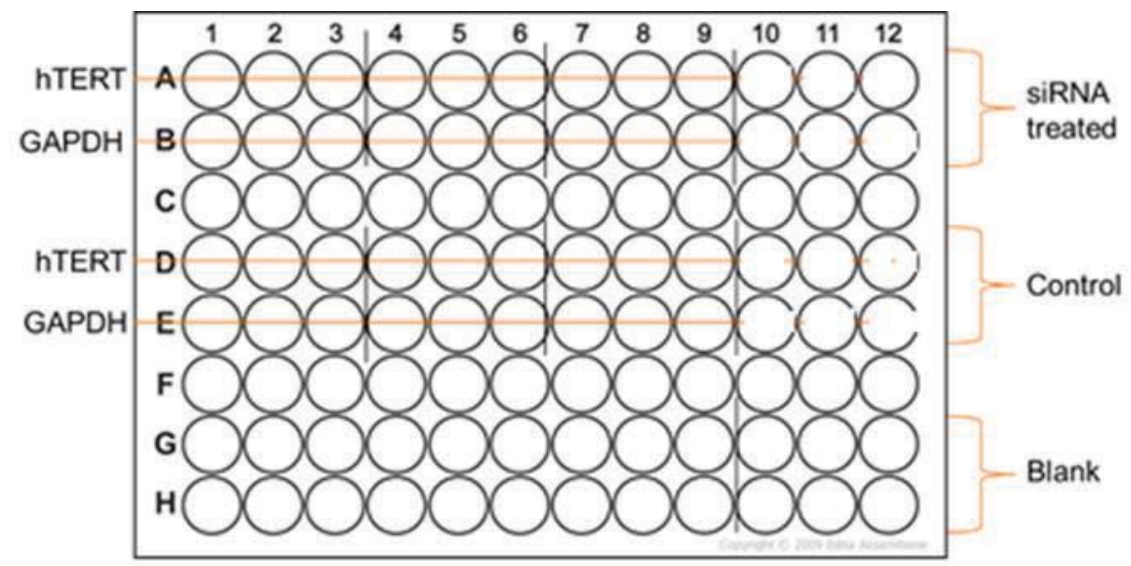

Figure 3. ELISA plate layout, each six square grouping (separated by vertical lines) corresponds to analyzed protein isolated from cells grown in one well of a six well plate.

protocol derived from Current Protocols in Molecular Biology by Ausubel and colleagues in 2003. ${ }^{18}$ Normalization of the ELISA absorbance values were performed as previously discussed with protocol published by Haimes and Kelley in $2013 .{ }^{17}$

\section{RESULTS}

The melt curve generated in this research (Figure 4) demonstrates that the PCR amplicons synthesized during the course of the reaction are from hTERT and GAPDH cDNA and that no detectable primer-dimers or non-specific amplifications occurred. The validity behind this conclusion can be found in the method by which this melting curve was generated. The final PCR solution is heated and each specific amplicon present within the solution will have a characteristic temperature at which it dissociates and therefore no longer fluoresces (due to the binding characteristics of SYBR ${ }^{\circledR}$ green). Due to this innate detachment temperature, each distinct amplicon will also produce a distinguishing peak on the melt curve. However, only two amplicons (one from hTERT and one from GAPDH) were synthesized during the course of the PCR reaction as validated by the two peaked melt curve. Without the melting curve data, it would be impossible to determine whether or not multiple, non-target amplicons were generated. Such an occurrence would engender false RNA quantification results. The data revealed that the siRNA treated cells possessed 97.8 percent less telomerase mRNA than the control group (Figure 5). Following ELISA absorbance normalization (as discussed above) a 53.1 percent protein knockdown was determined (Figure 6). Therefore, the siRNA treated cells possessed nearly fifty-three percent less telomerase protein than the untreated control cells. 


\section{DISCUSSION}

The 97.8 percent mRNA knockdown ( $\mathrm{p}$-value of 0.00768 , generated via t-test) attained in this investigation suggests that the transfection mechanism employed was relatively effective, supporting the original hypothesis. Which stated: siRNA can elicit the degradation of hTERT mRNA in cultured HeLa cells and this degradation will significantly reduce hTERT protein concentrations. Nonetheless, only a knockdown percentage of one hundred percent can effectively engender cancer cell crisis and in turn cell death. The efficacy of this PCR procedure is consistent with that determined by Deshayes and colleagues in $2008 .{ }^{13}$ Deshayes demonstrated that a specific domain in the MPG peptide, known as NLS, executes nucleotide binding. As the NLS domain was mutated, MPG's nucleotide binding ability was cooperatively modified. Thusly, the hTERT knockdown percentage may be fortified by manipulating the NLS domain structure. Additionally, their research discovered that the nucleotide delivery compartment (cytoplasm or nucleus) could be selectively chosen with certain NLS mutations. This occurrence advocates the fact that the NLS domain plays not only a role in nucleotide binding but a critical function in dictating the site at which the nanoparticles nucleotide passenger will depart. The MPG nanoparticle and its inclusive siRNA associate have been shown to participate in not only translational inhibition but transcriptional inhibition. The presence of a promoter binding sequence in a siRNA fragment may allow promoter synergy and consequently effective inhibition of gene transcription. A much more efficient, dual layered anti-cancer therapy could be introduced by utilizing such transcriptional and translational inhibition. This dual layered

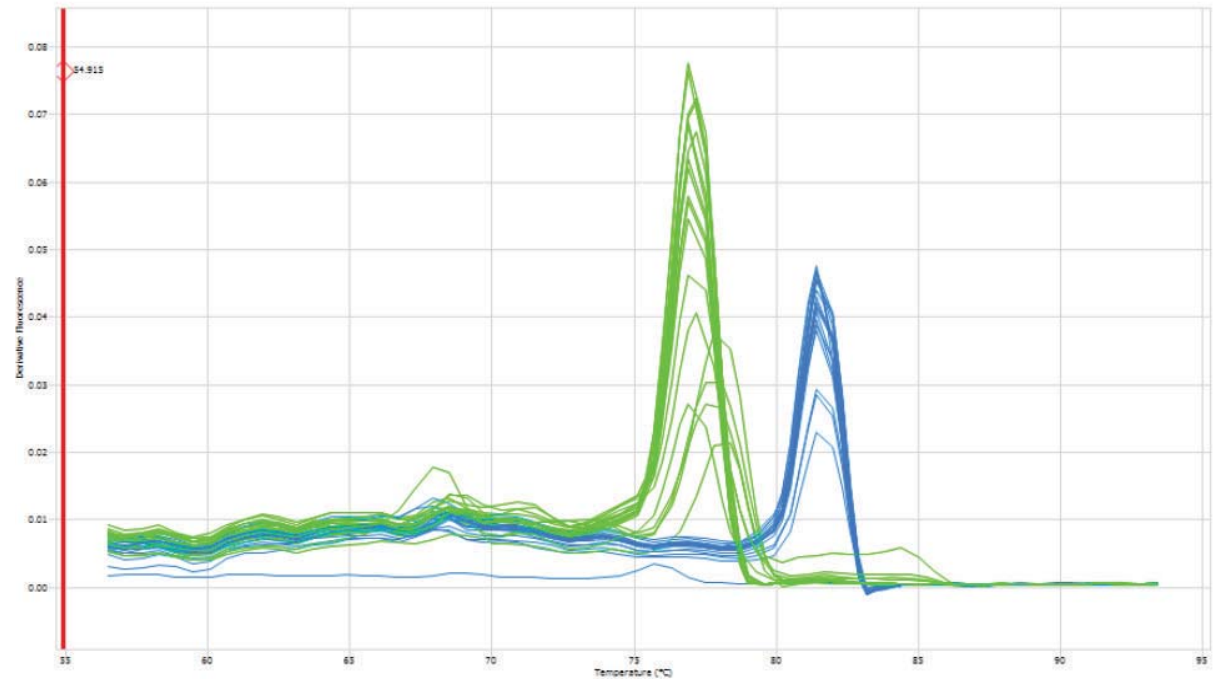

Figure 4. Melt Curve indicating PCR efficiency, twin peaks indicate that only two amplicons (GAPDH and hTERT) were generated in the reaction.

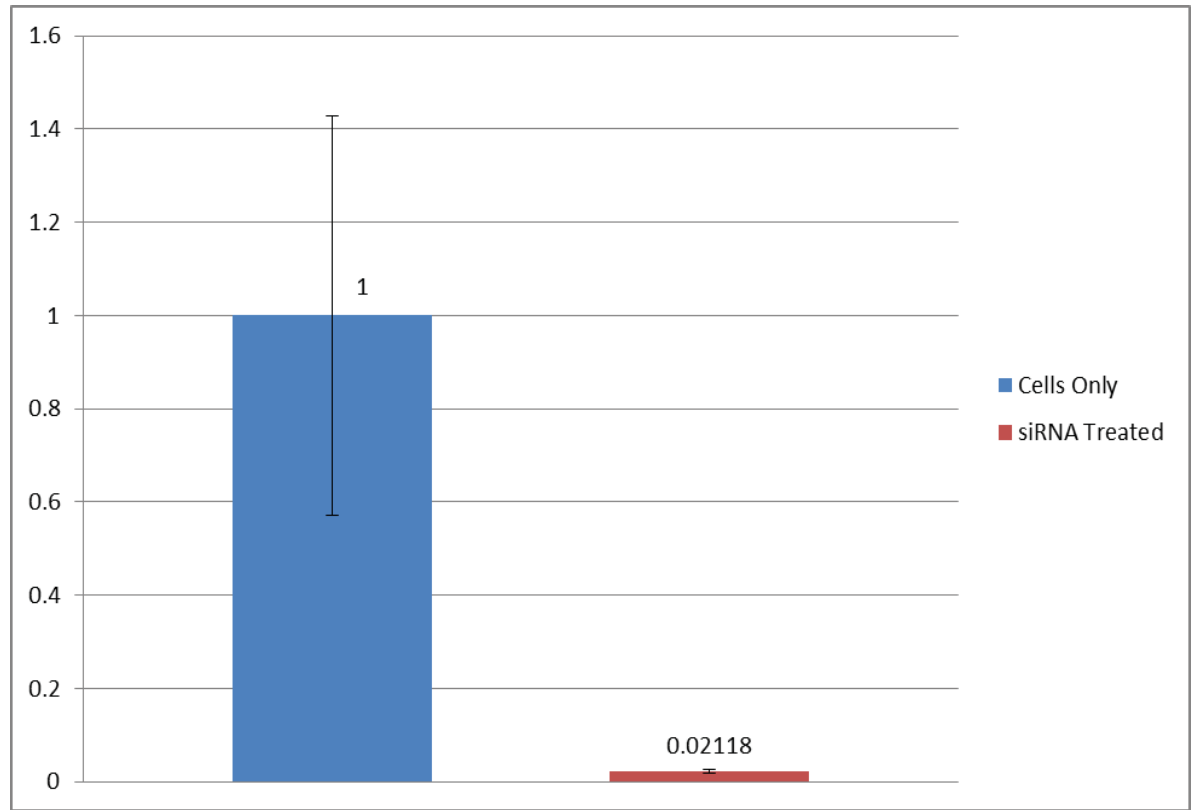

Figure 5. hTERT mRNA knockdown comparing control cells (set to $1 \mathrm{AU}$ ) to siRNA treated cells. 
anticancer therapy has the potential to augment the hTERT knockdown to one-hundred percent and thus be employed in anticancer clinical applications. The fifty-three percent protein knockdown, at first glance, may seem like an enormous aberration from the previously discussed PCR results. However, this knockdown value is consistent with previously published experimental data. Yi and colleagues in 2001 demonstrated that hTERT possesses a half-life value of around twenty-four hours; because the siRNA that was incorporated into the experimental cells does not directly induce telomerase protein degradation, those proteins present prior to siRNA exposure will still be functioning. ${ }^{19}$ However, throughout the course of the twenty-four hour incubation period with the small interference RNA, half or fifty percent of the hTERT protein will have degraded as exemplified by the 24hour half-life of the hTERT protein. Consequently, the 53.1 percent knockdown is consistent with the experimentally determined half-life value of the telomerase protein. The protein knockdown results presented herein is novel within the recently published research basin. The few published articles concerning hTERT protein knockdown via siRNA do correlate well with these results. For example, Xu and colleagues demonstrated that certain siRNA sequences can significantly reduce both the detectable hTERT mRNA and protein content of analyzed cells. ${ }^{20}$

\section{Conclusion}

A few conclusions can be gleaned from the results of the PCR analysis, the first of which is that siRNA can effectively trigger degradation of a particular mRNA and potentially destroy that proteins activity in the cell. This research also demonstrates that target proteins, not just the mRNA, are greatly reduced. In this case, any possible increase in translation of the TERT mRNA, if it occurred at all, did not offset the dramatic decrease in template available for translation. Consequently, if continual siRNA

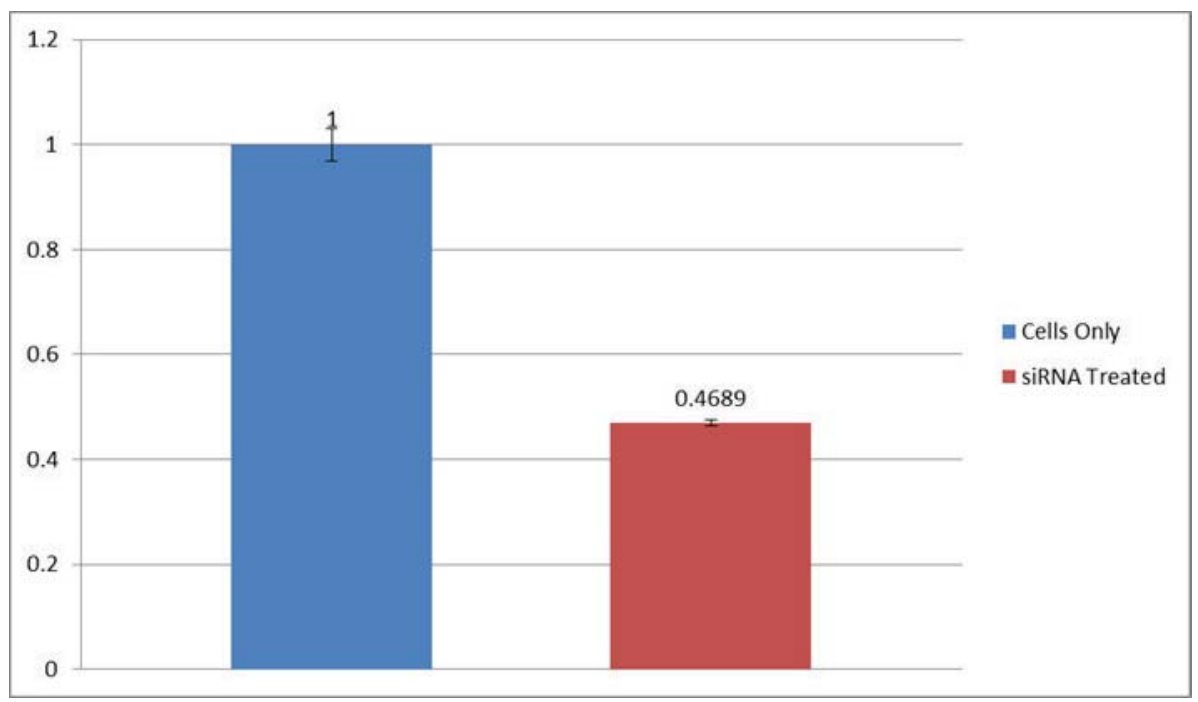

Figure 6. hTERT protein knockdown comparing control cells (set to 1 AU) vs. siRNA treated cells.

expression could be achieved, the telomerase content of a subjected cancer cell may eventually reach negligible levels at which point telomere elongation will no longer occur and crisis could soon follow. This research demonstrates that one potential mechanism by which perpetual siRNA expression and subsequent TERT protein knockdown may be achieved is with the use of h'TERT targeting, siRNA.

\section{Further Research}

The inclusion of an assay measuring cell proliferation, such as an MTT (3-(4,5-dimethylthiazol-2-yl)-2,5-diphenyltetrazolium bromide) between treated and untreated cells would substantiate the aforementioned claim of hTERT siRNA's ability to inhibit cancer cell proliferation. Such a project will be conducted in future research. Additionally, the author understands that a significant hurdle to effective treatment in a living system will likely be the continual delivery of siRNA to target cells (while avoiding non-targeted delivery). Continued research is warranted in both nanoparticle delivery as well as potential use of viral delivery systems tied to gene therapy.

\section{ACKNOWLEDGMENTS}

This research was supported in part by Dr. Douglas Christensen (Wayne State College), who advised the researcher and provided overall support for the project. A special thanks is also extended to Dr. Shawn Pearcy (Wayne State College) for his support with HeLa cell growth. Finally, I'd like to thank Dr. Gustavo Zardeneta for his assistance in Western Blot and ELISA protocol. 
Funding

This research was made possible by grants from the National Center for Research Resources (5P20RR016469) and the National Institute for General Medical Science (NIGMS) (8P20GM103427), a component of the National Institutes of Health (NIH) and its contents are the sole responsibility of the authors and do not necessarily represent the official views of NIGMS or NIH.

\section{REFERENCES}

1. Hanahan, D., Weinberg, R. (2011) Hallmarks of Cancer: The Next Generation, Cell 144, 646-674.

2. Hahn, W. (2003) Role of Telomeres and Telomerase in the Pathogenesis of Human Cancer, J Clin Oncol 21, $2034-2043$.

3. Olovnikov, A. (1996) Telomeres, telomerase, and aging: Origin of the theory, Exp Geront 31(4), 443-448.

4. Rodier, F., Campisi, J. (2011) Four faces of cellular senescence, J Cell Biol 192(4), 547-556.

5. Blasco, M. (2002) Telomerase beyond telomeres, Nat. Rev. Cancer 2, 627-633.

6. Saretzki, G., Sitte, N., Merkel, U., Wurm, R.E., von Zglinicki, T. (1999) Telomere shortening triggers a p53-dependent cell cycle arrest via accumulation of G-rich single stranded DNA fragments, Oncogene 18(37), 5148-5159.

7. Janknecht, R. (2004) On the road to immortality: hTERT upregulation in cancer cells, FEBS Lett 564(1-2), 9-13.

8. Bryan, T., Englezou, A., Dalla-Pozza, L., Dunham, M., Reddel, R. (1997) Evidence for an alternative mechanism for maintaining telomere length in human tumors and tumor-derived cell lines, Nat Med 3, $1271-1274$.

9. Sela, M. (1969) Antigenicity: Some Molecular Aspects, Science 166(3911), 1365-1374.

10. Bartel, D.P. (2004) MicroRNAs: genomics, biogenesis, mechanism, and function, Cell 116, 281-297.

11. Zeng, Y., Cullen, B.R. (2003) Sequence requirements for micro RNA processing and function in human cells, RNA 9, 112123.

12. Carthew, R.W., Sontheimer, E.J. (2009) Origins and Mechanisms of miRNAs and siRNAs, Cell 136(4), $642-655$.

13. Deshayes, S., Morris, M., Heitz, F., Divita, G. (2008) Delivery of proteins and nucleic acids using a non-covalent peptidebased strategy, Adv Drug Deliv Rev 60, 537-547.

14. Whitehead, K., Langer, R., Anderson, D. (2009) Knocking down barriers: advances in siRNA delivery, Nat. Rev. Drug Discovery 8(2), 129-138.

15. Reynolds, A., Leake, D., Boese, Q., Scaringe, S., Marshall, W.S., Khvorova, A. (2004) Rational siRNA design for RNA interference, Nat Biotechnol 22(3), 326-330.

16. Singh, V., Kumar, A. (2001) PCR Primer Design, Mol Bio Today 2(2), 27-32.

17. Haimes, J., Kelley, M. (2013) Demonstration of a $\Delta \Delta$ Cq Calculation Method to Compute Thermo Scientific Relative Gene Expression from qPCR Data. Lafayette, CO: Thermo Scientific.

18. Ausubel, F.M., Brent, R., Kingston, R.E., Moore, D.D., Seidman, J.G., Smith, J.A., Struhl, K. (2003) Current Protocols in Molecular Biology, John Wiley \& Sons Inc. 4648.

19. Yi, X., Shay, J.W., Wrighta, W.E. (2001) Quantitation of telomerase components and hTERT mRNA splicing patterns in immortal human cells, Nucleic Acids Res 29(23), 4818-4825.

20. Xu, H., Gong, X., Zhang, H., Zhang, Q., Zhao, D., Peng, J.X. (2015) Targeting Human Telomerase Reverse Transcriptase by a Simple siRNA Expression Cassette in HepG2 Cells, Hepat Mon 15(3), 24343.

\section{ABOUT THE STUDENT AUTHOR}

Shawn is a 2015 graduate of Wayne State College where he earned a double major Bachelors of Science degree in Biology and Chemistry. Shawn graduated Summa Cum Laude from WSC and hopes to attend graduate school for biochemistry in the fall of 2016. While attending Wayne State College, Shawn conducted a two year research project that he presented at the annual 2014 Nebraska Academy of Science spring conference in Lincoln, Nebraska.

\section{PRESS SUMMARY}

Cancer is one of the most important human diseases today, much of this danger is directly related to a cancer cells capability of the unrelenting division. This characteristic is conferred to the cell via reactivation of the telomerase gene and in turn the corresponding protein. If the method described herein could be developed into an in-vivo treatment procedure, such a process could be employed to target cancer cells for destruction. 